\title{
Non-specific inflammatory bowel diseases and the risk of tumour growth
}

\author{
EWA DUDZIŃSKA, MAGDALENA GRYZIŃSKA*, KATARZYNA OGNIK**, \\ MAGDALENA KRAUZE**, PIOTR LISTOS***
}

\author{
Department of Public Health, Faculty of Health Sciences, \\ Medical University of Lublin, W. Chodźki 1, 20-093 Lublin, Poland \\ *Department of Biological Basis of Animal Production, **Department of Biochemistry and Toxicology, \\ Faculty of Biology, Animal Sciences and Bioeconomy, University of Life Sciences in Lublin, \\ Akademicka 13, 20-950 Lublin, Poland \\ ***Department of Pathological Anatomy, Faculty of Veterinary Medicine, \\ University of Life Sciences in Lublin, Głęboka 30, 20-033 Lublin, Poland
}

Dudzińska E., Gryzińska M., Ognik K., Krauze M., Listos P.

Non-specific inflammatory bowel diseases and the risk of tumour growth

Summary

Inflammatory bowel disease (IBD), which includes mainly ulcerative colitis (UC) and Crohn's disease (Lesniowski-Crohn's, ChL-C, CD), is a chronic and recurrent inflammatory condition of the gastrointestinal tract with multifactorial causes. Both types of IBD are characterized by chronic inflammation with periods of remission and exacerbation. An increasing number of studies have recently shown that chronic inflammation plays an important role in the carcinogenesis of colorectal cancer (CRC), generating suitable microenvironments for the formation and progression of the disease. The main factors are chronic inflammation as well as the scope and duration of the disease. The pro-inflammatory interleukins IL-13, IL-8 and TNF- $\alpha$ play an important role in tumorigenesis. It is further emphasized that reactive oxygen species (ROS) and reactive nitrogen species produced by inflammatory cells may interact with key genes involved in carcinogenic pathways, such as TP53. Carcinogenesis in IBD involves proteins determined by the genes DLG5, OCTN and NOD2. Immunosuppressive drugs, such as thiopurines and methotrexate, may play a role in extra-intestinal tumour development by impairing the immune system and surveillance of tumour cells or by inducing DNA damage. Recognition of neoplastic changes associated with IBD is difficult due to the heterogeneity of the endoscopic image and variation in the diagnosis depending on the observer. Therefore surveillance of IBD patients by a multidisciplinary team is essential for early detection of the neoplastic process.

Keywords: non-specific inflammatory bowel disease, chronic inflammation, carcinogenesis

Inflammatory bowel disease (IBD), which includes mainly ulcerative colitis (UC) and Crohn's disease (Lesniowski-Crohn's, ChL-C, CD) in man, is a chronic and recurrent inflammation of the alimentary tract with multifactorial causes. Similar disorders are found in other animal species, and IBD has been observed in dogs and cats $(4,23)$. Causes of IBD include genetic susceptibility, environmental factors, bacterial agents, and damage to the epithelial barrier. Due to abnormal activation of the immune system and mucosal immune response, inflammatory reactions involving numerous immune cells and mediators are initiated (11). Both types of IBD are characterized by chronic inflammation with periods of remission and exacerbation. They may vary between mild and acute and often significantly affect the individual's quality of life. In 1952, Rosenberg and Crohn determined a connection between IBD (non-specific inflammatory bowel disease) and CRC (colorectal cancer). The CRC risk for patients suffering from IBD depends on the extent, duration and severity of the inflammation. The percentage for IBD-CRC associations is between $1 \%$ and $2 \%$ for all CRC units, which represents $10-15 \%$ of terminal cases of IBD (2). Most of sporadic colorectal tumours arise from adenomas, which are characterized primarily by epithelial dysplasia. A tumour developing in the case of IBD is usually invasive, poorly differentiated, multifocal and predisposed to location in the large intestine. The increased risk of CRC (colorectal cancer) in IBD (non-specific inflammatory bowel dis- 
ease) patients is associated with chronic inflammation of the intestinal epithelium. In most cases, neoplastic changes arise within inflammatory lesions during repair of the epithelium in the healing process of the intestinal mucosa (10).

The intestinal mucosa is an important defence system preventing induction of trauma by undigested substances, hydrochloric acid, ischaemia and infections. Every incident of damage to the mucosa is associated with a repair process, which is complex, but consists mainly of the immune response of granulation tissue formation, angiogenesis and epithelial regeneration. Such repair systems involve a potential risk of persistent intestinal inflammation, which may lead to chronic inflammation, as in IBD (25).

During inflammation, the fate of the cell depends on the balance between the pro- and anti-tumour immune response, and inflammation is currently thought to be associated with three stages of tumour growth: initiation, promotion and progression. Initiation includes a precancerous transformation of the cell. The inflammatory environment with a large number of cytokines and chemokines, as well as reactive nitrogen and oxygen species, leads to DNA mutation, epigenetic changes and genome instability, which may contribute to tumour initiation. The tumour promotion period includes the development of altered cells and inflammation, which is represented by apoptosis inhibition and faster angiogenesis and proliferation. This results in tumour progression and metastasis, leading to an increase in the size of the tumour, additional genetic changes and the spread of the tumour from its original site, which is also influenced by inflammation. Thus it is evident that there is a close link between inflammation and cancer in all stages of oncogenesis (7).

\section{Chronic inflammation in IBD and tumour development}

Crohn's patients whose disease is limited to the small intestine are included in the high risk group for CD-CRC, but there is a strong link between inflammation and CA-CRC (colitis-associated colorectal cancer). The risk of CRC in UC has been estimated at $2 \%$ after 10 years of the disease, $8 \%$ after 20 years and $18 \%$ after 30 years. Studies on UC-CRC have also noted a high concordance between the risk of CA-CRC and the location/extent of the disease. According to the standard incidence ratio (SIR), the incidence of CA-CRC is estimated at 1.7 for changes limited to the rectum, 2.8 for left-sided colitis and 14.8 for pancolitis (defined as extensive inflammation of the colon or affecting the entire large intestine). Studies on CD-CRC are complicated by the vast variation in the location of pathological changes in $\mathrm{CD}$. In the case of UC, the risk of CA-CRC depends on with the duration and acuteness of the disease. The CD-CRC relative risk (RR) is estimated at 2.9, 5.6 and 8.3 within 10,20 and 30 years of illness (12). An increasing number of studies have recently shown that chronic inflammation plays an important role in the carcinogenesis of CRC, generating suitable microenvironments for its formation and progression (22).

Patients with inflammatory bowel disease (IBD) have an increased risk of colorectal cancer (CRC). The main factors are chronic inflammation and the scope and duration of the disease. This increased risk of CRC is more probably associated with chronic inflammation of the intestinal mucosa than with genetic predisposition. Patients suffering from IBD for 8-10 years have an increased risk of dysplasia and CRC. Production of various inflammatory cytokines increases due to the penetration of immune cells in the intestinal mucosa of IBD patients. The balance between pro- and anti-inflammatory cytokines is considered to be the main pathogenesis factor of IBD, and the excessive production of inflammatory cytokines in IBD is linked to carcinogenesis (26).

IL-13 is an important factor in tumorigenesis. It may stimulate activation-induced cytidine deaminase (AID) as well as gastric inflammation with tumour cell generation. Moreover, IL-13 affects eosinophils, causing their long-term survival, activation and migration to inflammatory lesions. Previous reports suggest that IL-13 receptors undergo strong expression in intestinal epithelial cells. An elevated IL-13 level is also noted in the case of cancers of the large intestine, bladder, prostate and pancreas. Studies have shown that polymorphic variants of the IL-13 genes may increase the risk of breast cancer and mastocytosis, and may also lead to tumour growth and metastasis (21).

TLR signalling pathways are significant activating factors for NF-kB, making proinflammatory genes grow. Nevertheless, constant activation of these pathways also leads to such diseases as IBD and some cancers. It is well-documented that an elevated level of TNF- $\alpha$ is observed in the serum and small intestinal mucosa of IBD patients, whereas neutralization of TNF-a, which is the basis of anti-TNF therapy, is associated with improved health, particularly in Crohn's patients. TNF- $\alpha$ signals are transmitted by receptors, including two transmembrane receptors, TNFR1 and TNFR2. TNF- $\alpha$ signalling by TNFR1 and TNFR2 causes activation of transcription factors AP-1 and NF-kB, associated with cell proliferation, survival and/ or apoptosis. Increased TNFR2 expression is observed in cases of inflammation in IBD associated with tumour development. TNF- $\alpha$ and IL- 6 are thought to induce TNFR2 in colon cancer cells (19).

Reactive types of oxygen (ROS) and nitrogen generated by inflammatory cells may interact with the main carcinogenic gene groups, such as TP53. The wide group of factors inducing IBD also includes immune disorders causing abnormal stimulation of lymphocytes, macrophages or other immunocompetent cells. 
This leads to changes in cytokine expression, which may play a role in carcinogenesis. An increased level of pro-inflammatory mediators has been observed in the intestinal epithelium in both IBD and CRC. One of these is interleukin 8 (IL-8), a cytokine playing a key role in chemotaxis and migration of neutrophils, monocytes, lymphocytes and fibroblasts. IL-8 also regulates angiogenesis and proliferation of tumour cells. Research on CRC cells proves high expression of CXCR1 and CXCR2 receptors specific for 1L8 (21).

\section{Role of genetic factors leading to tumour development in IBD}

Long IBD duration increases the risk of intestinal cancer. Analysis of mutations in certain genes has shown that intestinal cancers developing in IBD patients differ from those occurring in patients without this disease. Intestinal tumours developing in patients with IBD have different genetic traits differentiating them from sporadic intestinal tumours (16). Sporadic CRC and CRC associated with IBD develop through different molecular carcinogenic pathways. Sporadic CRC may be the result of two important types of genetic chromosome instability (85\%) and microsatellite instability $(15 \%)$. Both of these mechanisms act in IBD-associated CRC, but at different moments and with different frequency (22). The initiation and development of CA-CRC (colitis-associated colorectal cancer) depend on the accumulation of mutations in various suppressor genes and oncogenes of intestinal tract epithelial groups of cells (12).

Tumour suppressor gene TP53 is located at $17 \mathrm{p} 13$ and encodes a transcription factor which regulates expression of genes involved in numerous biological processes, such as the control of the cell cycle, DNA repair and apoptosis. Mutations in TP53 have been reported in $50 \%$ of cases of CRC, and usually lead to the loss of transcriptional control. TP53 mutations mainly affect five hotspots: amino acid positions 175 , 245, 248, 273 and 282. Research has been conducted on the relationship between the polymorphism of codon 72 and cancer risk in IBD. It has shown that increased risk of CRC in IBD patients may be explained by an increase in the rate of TP53 mutations in patients with Arg 72 genotypes. However, further studies are needed to confirm the clinical significance of polymorphism and to better understand the effect of codon 72 polymorphism on the development of IBD (20). Genes of the inflammatory response underlying IBD, such as DLG5, OCTN and NOD2, hypothetically play a role in the progression of CRC. Among genes associated with IBD, genetic variants of NOD2/CARD15 have been studied most intensively in terms of their association with susceptibility to CRC (13).

Little is known of the development of progressive genetic changes in the intestinal epithelium in IBD, which progress from benign mucosa to dysplasia and finally to invasive CRC. Partial elucidation of this development may form the genetic basis for increased risk of CRC in IBD patients. In addition, the pathogenesis of IBD-CRC (inflammatory bowel diseaseassociated colorectal cancer) is poorly described in comparison with sporadic CRC. Interestingly, different mechanisms have been suggested for the two cases. For example, mutations of the geneTP53 in sporadic CRC appear at a late stage in the disease (between the late adenoma and cancer), whereas in IBD they occur at an earlier stage of the disease and lead to initiation of IBD-CRC (inflammatory bowel disease-associated colorectal cancer). MicroRNA (miRNA), which seems to play an important role in carcinogenesis, regulates the expression of the gene through binding with specific RNA (messenger RNA) and leads to degradation or suppression of protein production. Changes in the miRNA expression profile have been observed in many cancers. There is evidence that miRNA acts as oncogenic miRNA or a cancer suppressor. Abnormal miRNA expression has been shown to be linked to deregulation of TP53, an important tumour suppressor, whose activity is often lost in CRC (9).

\section{Other risk factors for tumour development in IBD}

The risk of tumour development in IBD is mainly ascribed to two factors: chronic inflammation and immunosuppression. Immunosuppressive drugs, such as thiopurines and methotrexate, may play an important role in parenteral tumour development through impairment of the immune system and the control of cancer cells or induction of DNA damage (Fig. 1). The potential risk of cancer is an important and growing concern associated with long-term immunosuppressive treatment of IBD, particularly in the context of the ageing IBD population. Various non-intestinal malignant tumours, such as lymphoproliferative disorders and non-melanoma skin cancer, are more frequent in IBD patients than in the general population, especially in those taking immunosuppressants. Furthermore, the risk of cancer of the urinary tract in IBD patients taking thiopurines seems to be elevated as well $(3,4)$. An increased risk of malignant tumours, including CRC, is often associated with primary sclerosing cholangitis (PSC), a chronic, progressive disease which is usually associated with $\operatorname{IBD}(8,17)$.

The pathogenesis of inflammatory bowel disease (IBD) involves a complex interaction between changes in numerous genes and abnormal interaction with environmental factors. There is increasing evidence that epigenetic factors may play a significant role in the pathogenesis of IBD. Considerable efforts have been made to discover genetic and epigenetic factors which may increase the risk of IBD and cancer. Tumorigenesis is a multi-stage process (initiation, promotion and progression) and is characterized by the accumulation of numerous modifications, includ- 


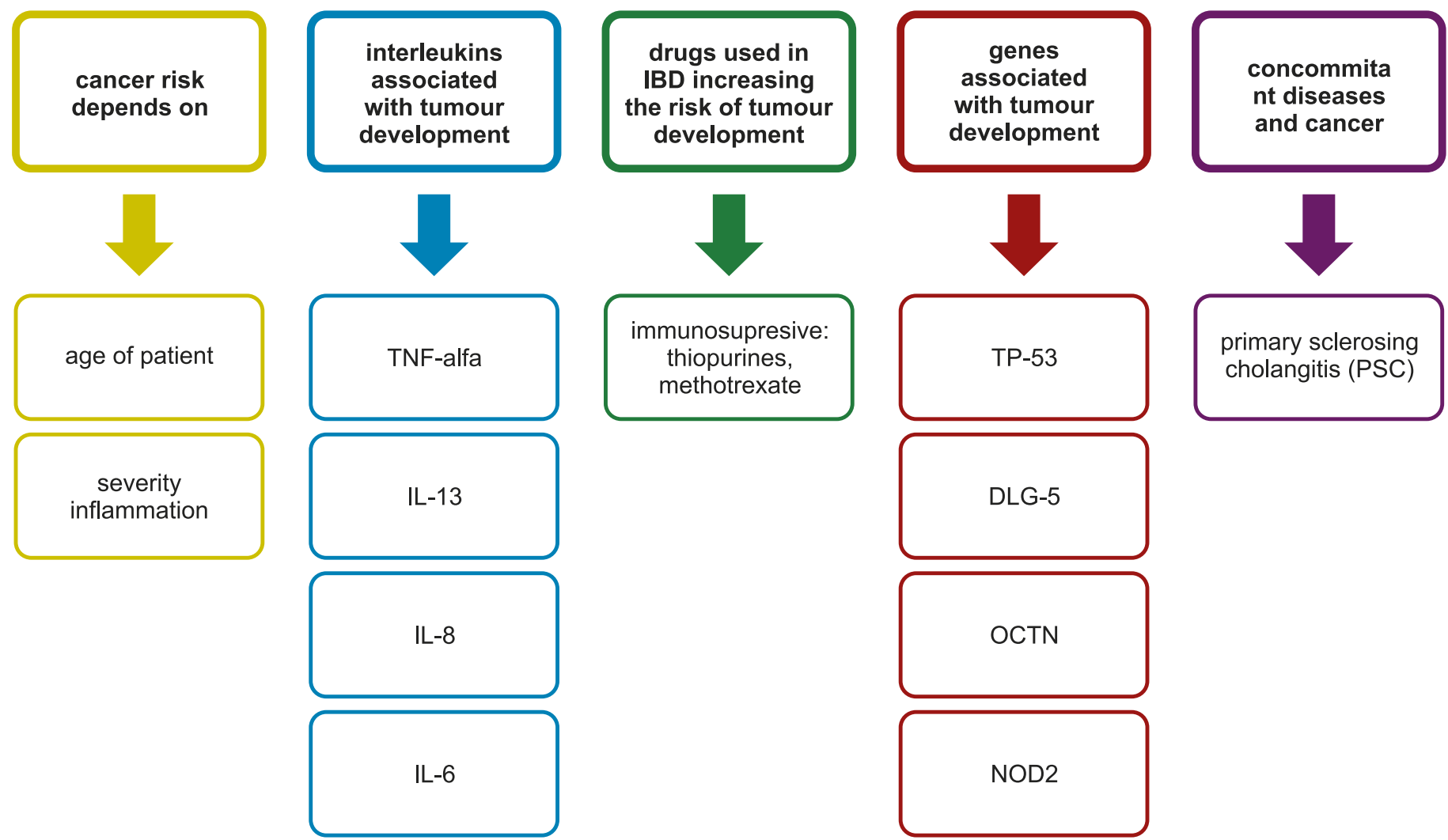

Fig. 1. Factors in parenteral tumour development

ing genetic, cytogenetic and epigenetic changes. More precisely, IBD may be caused by interactions between the host and the environment, which involve intestinal microflora, the immune system, and genetic determinants. Research has shown that the structure of the host organism and specific environmental factors, such as cigarette smoking, drug use, breastfeeding and diet, enter into interactions via epigenetic mechanisms, or more precisely DNA methylation $(6,24)$.

Chronic inflammation of the intestinal mucosa, parenteral symptoms and immunosuppressive treatment in IBD may increase the risk of colorectal cancer (CRC) (15). During the development of inflammatory bowel disease (IBD), inducible expression of numerous genes takes place, each of which has a unique effect, such as activation of the immune system, clearance of bacteria, or tissue repair or reconstruction. Deregulation or imbalance of any of these processes may contribute to the development of intestinal cancer (14).

The risk of carcinogenesis depends on the severity, scope and duration of IBD. It is recommended that patients have a colonoscopy with a biopsy every $1-2$ years after 8-10 years with the disease to detect dysplasia and rule out neoplasia (18). Diagnosis of neoplastic changes associated with IBD is difficult due to the heterogeneity of the endoscopic image and variation in the diagnosis depending on the observer. Established guidelines for surveillance of IBD patients are aimed at early detection of tumours. Authors emphasize that to optimize treatment effects and detect neoplastic changes at an early stage, a multidisciplinary team is needed, including a surgeon, gastroenterologist, pathologist and a patient who understands the character of the disease, including risks associated with cancer $(1,26)$.

It is important to be aware of the many factors that may play a role in the initiation of neoplastic changes in IBD. Therefore multidisciplinary medical care of the IBD patient and endoscopic monitoring of the state of the intestinal mucosa is essential, especially after several years of the disease.

\section{References}

1. Abraham B. P.: Cancer surveillance in ulcerative colitis and Crohn's disease: new strategies. Curr. Opin. Gastroenterol. 2016, 32, 32-37.

2. Althumairi A. A., Lazarev M. G., Gearhart S. L.: Inflammatory bowel disease associated neoplasia: A surgeon's perspective. World J. Gastroenterol. 2016, 22, 961-973

3. Biancone L., Onali S., Petruzziello C., Calabrese E., Pallone F.: Cancer and immunomodulators in inflammatory bowel diseases. Inflamm. Bowel. Dis. 2015, 21, 674-698.

4. Cerquetella M., Spaterna A., Laus F., Tesei B., Rossi G., Antonelli E., Villanacci $V$., Bassotti $G$.: Inflammatory bowel disease in the dog: Differences and similarities with humans. World J. Gastroenterol. 2010, 19, 1050-1056.

5. Derikx L. A., Nissen L. H., Drenth J. P., van Herpen C. M., Kievit W., Verhoeven R. H., Mulders P. F., Hulsbergen-van de Kaa C. A., Boers-Sonderen M. J., van den Heuvel T. R., Pierik M., Nagtegaal I. D., Hoentjen F.: Better survival of renal cell carcinoma in patients with inflammatory bowel disease. Oncotarget. 2015, 6, 38336-38347

6. Gryzinska M., Andraszek K., Jocek G.: DNA methylation analysis of the gene CDKN2B in Gallus gallus (Chicken). Folia Biologica (Kraków) 2013, 61, 165-171.

7. Hartnett L., Egan L. J.: Inflammation, DNA methylation and colitis-associated cancer. Carcinogenesis 2012, 33, 723-731.

8. Jones R., Scobey M., Cheng J.: The Risk of Colon Cancer in Inflammatory Bowel Disease. J. Gastroint. Dig. Syst. 2014, 4, 723-731.

9. Kanaan Z., Rai S. N., Eichenberger M. R., Barnes C., Dworkin A. M., Weller C., Cohen E., Roberts H., Keskey B., Petras R. E., Crawford N. P., Galandiuk S.: 
Differential micro RNA expression tracks neoplastic progression in inflammatory bowel disease-associated colorectal cancer. Hum. Mutat. 2012, 33, 551-560

10. Karczewski J., Mazur M., Rychlewska-Hańczewska A., Adamski Z.: Rola limfocytów Th17 w patogenezie raka jelita grubego. Postepy Hig. Med. Dosw. 2014, 68, 42-47.

11. Korolkova O. Y., Myers J. N., Pellom S. T., Wang L., M'Koma A. E. Characterization of Serum Cytokine Profile in Predominantly Colonic Inflammatory Bowel Disease to Delineate Ulcerative and Crohn's Colitides. Clin. Med. Insights Gastroenterol. 2015, 8, 29-44.

12. Kraak L. van Der, Gros P., Beauchemin N.: Colitis-associated colon cancer Is it in your genes? World J. Gastroenterol. 2015, 21, 11688-11699.

13. Lau T. P., Roslani A. C., Lian L. H., Lee P. C., Hilmi I., Goh K. L., Chua K. H.: NOD2/CARD15 variants in Malaysian patients with sporadic colorectal cancer. Genet. Mol. Res. 2014, 13, 7079-7085.

14. Low D., Subramaniam R., Lin L., Aomatsu T., Mizoguchi A., Ng A., DeGruttola A. K., Lee C. G., Elias J. A., Andoh A., Mino-Kenudson M., Mizoguchi E. Chitinase 3-like 1 induces survival and proliferation of intestinal epithelial cells during chronic inflammation and colitis-associated cancer by regulating S100A9. Oncotarget. 2015, 6, 36535-36550.

15. Meyer L., Simian D., Kronberg U., Estay C., Lubascher J., Figueroa C. Quera R.: Development of malignant tumors in patients with inflammatory bowel disease. Rev. Med. Chil. 2015, 143, 834-840.

16. Robles A. I., Traverso G., Zhang M., Roberts N. J., Khan M. A., Joseph C., Lauwers G. Y., Selaru F. M., Popoli M., Pittman M. E., Ke X., Hruban R. H., Meltzer S. J., Kinzler K. W., Vogelstein B., Harris C. C., Papadopoulos N. Whole-Exome Sequencing Analyses of Inflammatory Bowel Disease-Associated Colorectal Cancers. Gastroenterology 2016, 150, 931-943.

17. Rossi R. E., Conte D., Massironi S.: Primary sclerosing cholangitis associated with inflammatory bowel disease: an update. Eur. J. Gastroenterol. Hepatol. 2016, 28, 123-131
18. Rubin D. C., Shaker A., Levin M. S.: Chronic intestinal inflammation: inflammatory bowel disease and colitis-associated colon cancer. Front. Immunol. 2012, 3, 107 .

19. Thagia I., Shaw E. J., Smith E., Else K. J., Rigby R. J.: Intestinal epithelial suppressor of cytokine signaling 3 enhances microbial-induced inflammatory tumor necrosis factor- $\alpha$, contributing to epithelial barrier dysfunction. Am. J. Physiol. Gastrointest. Liver Physiol. 2015, 308, 25-31.

20. Volodko N., Salla M., Eksteen B., Fedorak R. N., Huynh H. O., Baksh S. TP53 codon $72 \mathrm{Arg} / \mathrm{Arg}$ polymorphism is associated with a higher risk for inflammatory bowel disease development. World J. Gastroenterol. 2015, 21, 10358-10366

21. Walczak A., Przybylowska K., Dziki L., Sygut A., Chojnacki C., Chojnacki J., Dziki A., Majsterek I.: The 1L-8 and IL-13 Gene Polymorphisms in Inflammatory Bowel Disease and Colorectal Cancer. DNA Cell. Biol. 2012, 31, 1431-1438.

22. Wang Z. H., Fang J. Y.: Colorectal Cancer in Inflammatory Bowel Disease: Epidemiology, Pathogenesis and Surveillance. Gastrointest. Tumors. 2014, 1, 146-154.

23. Willard M. D.: Feline inflammatory bowel disease: a review. J. Feline Med Surg. 1999, 1, 155-164.

24. Yi J. M., Kim T. O.: Epigenetic alterations in inflammatory bowel disease and cancer. Intest. Res. 2015, 13, 112-121.

25. Yoshimi K., Tanaka T., Serikawa T., Kuramoto T.: Tumor suppressor APC protein is essential in mucosal repair from colonic inflammation through angiogenesis. Am. J. Pathol. 2013, 182, 1263-1274.

26. Zhiqin W., Palaniappan S., Raja Ali R. A.: Inflammatory Bowel Disease-related Colorectal Cancer in the Asia-Pacific Region: Past, Present, and Future. Intest. Res. 2014, 12, 194-204.

Corresponding author: Piotr Listos DVM, PhD, Głęboka 30, 20-612

Lublin, Poland; e-mail: piotr.listos@up.lublin.pl 